\title{
A Study of Echocardiographic Changes in Patients with Newly Diagnosed Primary Hypothyroidism: A Cross-sectional Study
}

\section{Prabin Khatri ${ }^{*}$, Suman Sapkota ${ }^{1}$, Aryan Neupane ${ }^{2}$, Narayan Gautam $^{3}$, Saphal Nath Subedi², Ashish Banjade ${ }^{2}$, Smile Kharel²}

\section{Author Info:}

${ }^{1}$ Department of Internal Medicine, Universal College of Medical Sciences- Teaching Hospital, Bhairahawa, Nepal

2Intern, Universal College of Medical Sciences- Teaching Hospital, Bhairahawa, Nepal 3Department of Biochemistry, Universal College of Medical Sciences Teaching Hospital, Bhairahawa, Nepal

*Corresponding Author:

Dr. Prabin Khatri, Associate Professor, Department of Internal Medicine, Universal College of Medical Sciences

\section{Email/Contact:}

prabinkhatri@hotmail.com 977-9851176799

\section{ABSTRACT}

Background: Hypothyroidism is known to cause reversible cardiac dysfunction in humans. Thyroid hormone alters the cardiovascular hemodynamics. This study was performed to assess echocardiographic changes in untreated newly diagnosed primary hypothyroid states as such studies are scarce in our setting.

Methods: This hospital based cross-sectional study was conducted among 71 patients with newly diagnosed primary hypothyroidism who presented to the medicine outpatient department, at Universal College of Medical Sciences and Teaching Hospital (UCMS-TH), Bhairahawa, Nepal from December 2018 to June 2020 after taking ethical clearance from institutional review committee (UCMS/ IRC/212/18). Thyroid function test and echocardiography were carried out in patients under study. The data was analyzed with SPSS Version 16.

Results: A total of 71 patients (52 females and 19 males) with primary hypothyroidism were enrolled for the study. Among these 58 cases were overt/clinical hypothyroidism, and 13 cases were subclinical hypothyroidism. Echocardiography was abnormal in $63.4 \%$ of cases with $43.7 \%$ showing diastolic dysfunction, $18.3 \%$ diastolic interventricular septal thickness abnormality, $8.5 \%$ diastolic left ventricular posterior wall thickness abnormality, and $8.4 \%$ pericardial effusion.

Conclusion: Our study found diastolic dysfunction, diastolic interventricular septal thickness abnormality, diastolic left ventricular posterior wall thickness abnormality, and pericardial effusion as the common echocardiographic change in patients with newly diagnosed primary hypothyroidism. Therefore, our study highlights the need for cardiac evaluation of patients with primary hypothyroidism so that early intervention can be performed to improve the clinical outcome.

Keywords: Primary hypothyroidism, Echocardiography, Diastolic dysfunction.

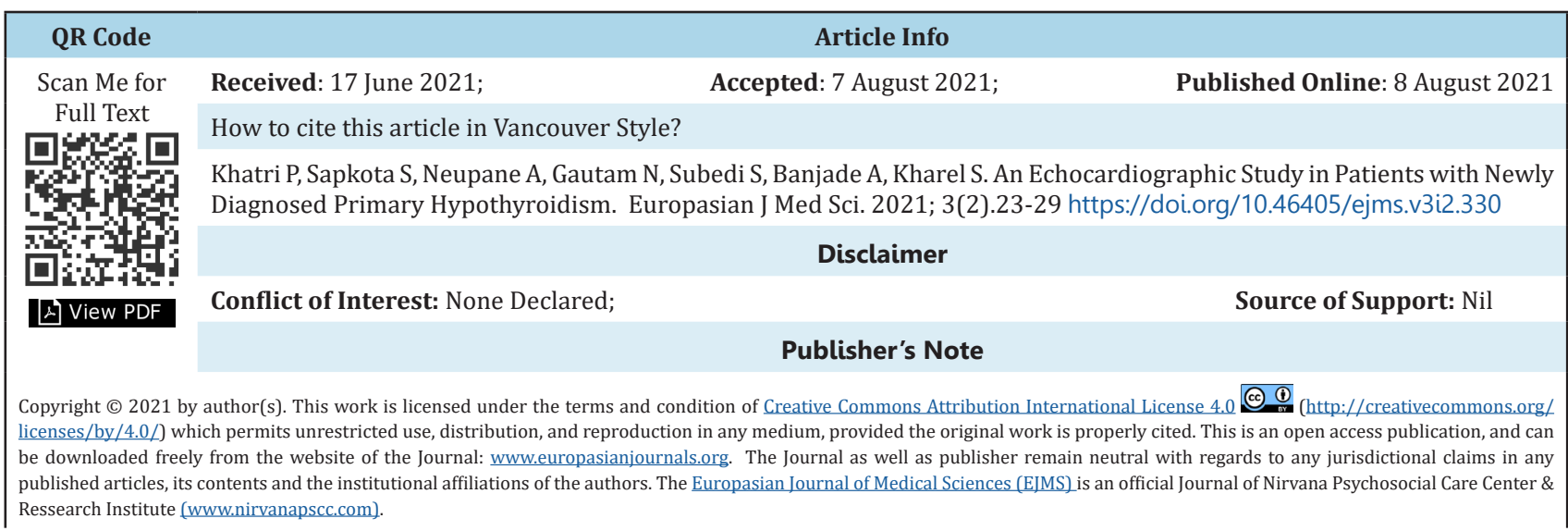




\section{INTRODUCTION}

Thyroid dysfunction is a common endocrine disorder affecting about 300 million people worldwide with over half estimated to be unaware of their condition. ${ }^{1}$ In Nepal, the prevalence of thyroid disorders in the general population is about $4.32 \% .{ }^{2}$ Hypothyroidism is a clinical syndrome which results in deficiency of thyroid hormone. In primary hypothyroidism, there is a defect within the thyroid gland and in secondary hypothyroidism, indirect pathologies (disorders of hypothalamus or pituitary gland) contribute to the decrease in circulating hormone levels. ${ }^{3}$ Overt hypothyroidism refers to cases in which the serum thyroid stimulating hormone (TSH) concentration is elevated and serum FT4 (free tetraidothyroxine) is below the reference range. Subclinical hypothyroidism is defined as a serum TSH above the defined upper limit of the reference range, with a serum FT4 within the reference range. ${ }^{4}$

Thyroid hormone has the ability to alter the cardiovascular hemodynamics. Besides its direct effects, the thyroid hormone also causes a secondary increase in cardiac contractility by increasing the substrate requirement and oxygen consumption. ${ }^{5}$ Thyroid hormone is known to increase sympathetic activity, angiotensinogen and atrial natriuretic peptide. It decreases vasopressin. ${ }^{6}$ Hypothyroidism impairs the relaxation of the vascular smooth muscle, produces arterial stiffness and also decreases endothelial nitric oxide which leads to increased vascular resistance (leading to an increase in diastolic BP). ${ }^{7}$ Patients with severe and longstanding hypothyroidism can develop pericardial effusions. Electrocardiogram (ECG) changes due to hypothyroidism present as sinus bradycardia, low amplitude P wave, QRS complex, prolonged action potential, PR interval, QT interval and ST segment alteration. ${ }^{8}$ Various studies have found links between sub-clinical hypothyroidism, ischemic heart disease and cardiovascular mortality. ${ }^{7}$ Echocardiography findings in hypothyroidism ranged from pericardial effusion, diastolic dysfunction, interventricular septal thickening, reduced ejection fraction and dilated cardiomyopathy. ${ }^{9}$ Being a non-invasive method, echocardiography can play an important role in recognition of the cardiac pathology and follow up effect of the therapy. ${ }^{10}$ This study was performed to assess echocardiographic changes in untreated newly diagnosed primary hypothyroid states.

\section{MATERIALS AND METHODS}

This study was conducted in Universal College of Medical Sciences Teaching Hospital, Bhairahawa, Nepal in the context of newly diagnosed primary hypothyroidism. Case screening for cardiovascular manifestations with echocardiography was performed at the time of diagnosis of primary hypothyroidism. Study was conducted for one and half year's duration from 9th December 2018 to 30th June 2020. It is a cross sectional observational study carried out on patients with newly diagnosed case of primary hypothyroidism visiting outpatient department of internal medicine at UCMS-TH.

Sample size was calculated by using the formula. N $=(\mathrm{Z} 2 \mathrm{PQ}) / \mathrm{d} 2$, where, $\mathrm{Z}=1.96$ for 95\% Reliability; $\mathrm{P}=$ Estimated proportion 0.16 (from study done by aryal et.al). ${ }^{11}$ and $d=$ Maximum tolerance error $=0.1$. Considering a $10 \%$ attrition rate, a minimum of 58 patients were required for the study. In our study 71 newly diagnosed cases of primary hypothyroidism were enrolled.

All newly diagnosed cases of primary hypothyroidism presenting to the OPD of Internal Medicine in Universal College of Medical Sciences\& Teaching Hospital (UCMSTH) age 18 years and above were included in the study. Whereas, patients below 18 years of age, patients with secondary hypothyroidism, patients with underlying heart disease, chronic obstructive pulmonary disease, severe anemia, diabetes mellitus or any other endocrine disorder, patients taking beta blockers, lithium, oral contraceptive pills, steroids, amiodarone, and those consuming alcohol, patients refusing participation in the study and pregnant patients were excluded from the study.

Investigations: Thyroid function test was measured using vitro chemiluminescent immunoassay for quantitative determination of free T3 (fT3), T4 and TSH in human serum using the MAGLUMI series fully auto chemiluminescence immunoassay analyzer. Thyroid hormone abnormalities were considered if patients' thyroid hormone levels were outside the normal values; fT3 (2.0-4.2 pg/ml), fT4 (8.9-17.2 pg/ml) and TSH (0.3$4.5 \mathrm{mIU} / \mathrm{ml})$.

Echocardiographic studies were done with a twodimensional (2-D) echocardiography machine GE VIVD-3 with 2 Megahertz (MHz) transducer probe. All studies were performed during normal quiet respiration. Two dimensional and M-mode tracing were obtained in the parasternal window in the slight left lateral position. Five consecutive sinus beats were measured and averaged. Cursor-derived M-mode recordings obtained during two dimensional echocardiographic examinations were used to validate electronic measurements.

Data Management: The data were collected and entered in the proforma for each patient by the investigator. The data collected were entered in Statistical Package for Social Sciences (SPSS) Version 16.

Categorical data were expressed as frequencies and corresponding percentage. Quantitative variables were 
presented as mean ( \pm standard deviation). Independent t-test, Paired t-test were applied to test the significant difference between quantitative and categorical variables and chi-square test was applied to test the significant difference between two categorical variables. Statistical significance was determined at 5\% level of significance.

Ethical Clearance: The permission to conduct this research was taken from the institutional review committee of UCMS-TH (UCMS/IRC/212/18) on $18^{\text {th }}$ December 2018. Written informed consent was obtained from all the participants.

\section{RESULTS}

Out of 71 newly diagnosed primary hypothyroid patients, $73.2 \%$ are female and $26.8 \%$ are male. The median age was 45 years for all primary hypothyroidism, subclinical and overt hypothyroidism patients. The results showed that both subclinical and overt hypothyroidism was mostly seen in the age group 36-45 years and was least in the 18-25 years age group. This is shown in Figure 1. The association between age group and subclinical and overt hypothyroidism was found to be statistically insignificant $(\mathrm{p}=0.87)$. The bivariate logistic regression showed that age group 36-45 were about 1.67 times more likely to have subclinical hypothyroidism as compared to age group $18-25(\mathrm{OR}=1.67,95 \% \mathrm{CI}=0.15$ $17.89, \mathrm{p}=0.67)$ and age group $35-45$ were two times more likely to have overt hypothyroidism as compared to age group $18-25$ years $(\mathrm{OR}=2.0,95 \% \mathrm{CI}=0.10$ 39.07, $\mathrm{p}=0.65$ ).

Regarding presenting complaint in the study, as shown in Table1, the most common was constipation (50.70\%) followed by weight gain (42.30\%). Other symptoms were lethargy $(39.40 \%)$, and dyspnea $(23.90 \%)$. Few cases $(19.70 \%)$ presented with dry skin.

As depicted in Table 2, the mean TSH value of subclinical and overt cases was $8.73 \& 41.72$ respectively with a mean difference of 27.42 and the mean difference was found to be statistically significant $(\mathrm{P}<0.001)$. It can be concluded that the mean TSH value of overt hypothyroidism was significantly high. Similarly, there was significant mean difference in T3 \& T4 value between Subclinical and Overt hypothyroidism. T3 and T4 were found significantly high in overt hypothyroidism.

The prevalence of abnormal echocardiographic findings was found to be $63.4 \%(45 / 71)$ for hypothyroid patients with $67.2 \%(39 / 58)$ in overt and 46.2\% (6/13) in subclinical hypothyroidism. As shown in Table 4, the odds ratio of overt hypothyroidism for different Echocardiographic changes are ejection fraction $(\mathrm{OR}=0.09, \quad 95 \% \quad \mathrm{CI}=0.08-1.16, \mathrm{p}=0.065)$, diastolic interventricular septal thickness $(\mathrm{OR}=0.62, \quad 95 \%$ $\mathrm{CI}=0.16-2.99, \mathrm{p}=0.62)$, pericardial effusion $(\mathrm{OR}=1.13$,

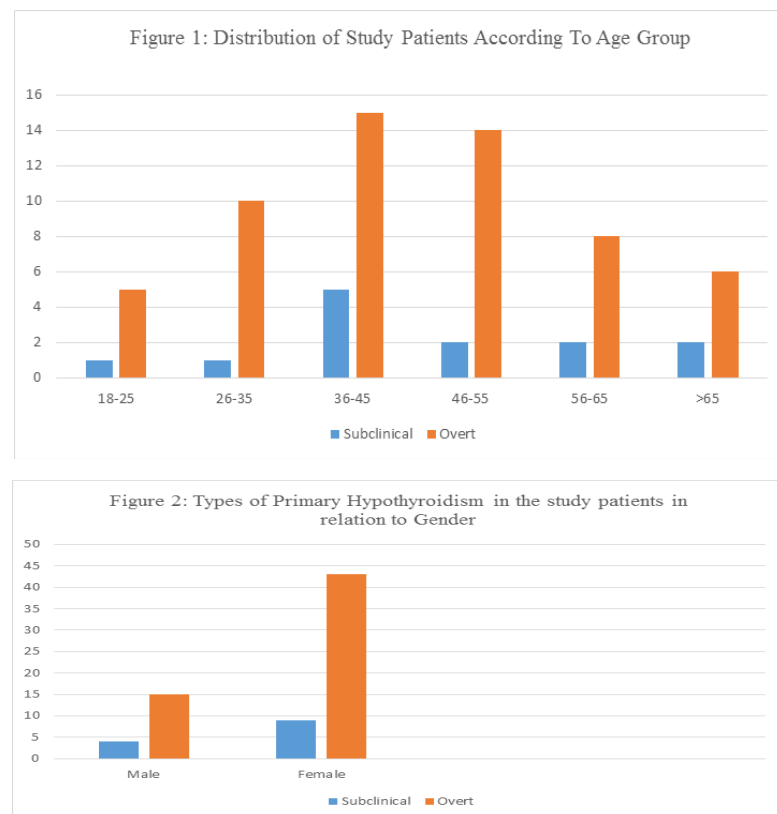

95\% $\mathrm{CI}=0.12-10.59, \mathrm{p}=0.91)$, and diastolic dysfunction $(\mathrm{OR}=12.85,95 \% \mathrm{C} .=1.57-105.41, \mathrm{p}=0.017)$. The odds ratio of subclinical hypothyroidism for different echocardiographic changes are ejection fraction $(\mathrm{OR}=10.36,95 \% \mathrm{CI}=0.86-124.46, \mathrm{p}=0.065)$, diastolic interventricular septal thickness $(\mathrm{OR}=1.14, \quad 95 \%$ $\mathrm{CI}=0.33-6.19, \mathrm{p}=0.62)$, pericardial effusion $(\mathrm{OR}=0.88$, $95 \% \mathrm{CI}=0.09-8.27, \mathrm{p}=0.91)$, and diastolic dysfunction $(\mathrm{OR}=12.85,95 \% \mathrm{CI}=1.57-105.41, \mathrm{p}=0.04)$.

\section{DISCUSSION}

In this study, echocardiographic changes were studied in newly diagnosed primary hypothyroid patients attending outpatient department at our center. A total of 71 cases were enrolled in the study including subclinical and Table 1: Distribution of Study Patients According to Clinical Presentation

\begin{tabular}{lll}
\hline Presenting Complaints & Frequency & Percentage \\
\hline Constipation & 36 & $50.70 \%$ \\
Weight Gain & 30 & $42.30 \%$ \\
Lethargy & 28 & $39.40 \%$ \\
Dyspnea & 17 & $23.90 \%$ \\
Cold Intolerance & 17 & $23.90 \%$ \\
Menstrual & 16 & $22.50 \%$ \\
Irregularities & & \\
Dry Skin & 14 & $19.70 \%$ \\
\hline
\end{tabular}

overt hypothyroidism who presented for the first time without any known co-morbidities and medication use.

In this study among randomly selected 71 cases, 19 cases $(26.8 \%)$ were males and 52 cases $(73.3 \%)$ female. A study by Mahato RV et al. in 2015 in Tribhuvan University Teaching hospital also had 
Table 2: Distribution of Study Patients with Respect to Thyroid Function Test

\begin{tabular}{ccccccc}
\hline Thyroid function test & Number (n) & Mean & Standard Deviation (SD) & Mean Difference & p-value \\
\hline \multirow{2}{*}{ T3 } & Subclinical & 13 & 2.37 & 1.05 & 0.61 & .088 \\
& Overt & 58 & 1.76 & 1.40 & & \\
\multirow{2}{*}{ T4 } & Subclinical & 13 & 9.56 & 1.56 & 5.30 & $<0.001$ \\
& Overt & 58 & 4.27 & 2.10 & & \\
\multirow{2}{*}{ TSH } & Subclinical & 13 & 8.73 & 0.65 & 33.99 & $<0.001$ \\
& Overt & 58 & 41.72 & 31.89 & & \\
\hline
\end{tabular}

Table 3: Association of ECHO Abnormality in Relation to Types of Primary Hypothyroidism

\begin{tabular}{|l|l|l|l|l|l|}
\hline \multirow{2}{*}{ Echo } & \multicolumn{2}{|l|}{ Hypothyroidism } & \multirow{2}{*}{ Total } & Chi-Square & p value \\
\cline { 2 - 4 } & Subclinical & Overt & & \\
\hline Normal & $7(53.8 \%)$ & $19(32.8 \%)$ & $26(36.6 \%)$ & & \multirow{2}{*}{0.2} \\
\cline { 1 - 4 } Abnormal & $6(46.2 \%)$ & $39(67.2 \%)$ & $45(63.4 \%)$ & & \\
\hline Total & 13 & 58 & 71 & \\
\hline
\end{tabular}

similar sex distribution. ${ }^{12}$ Higher prevalence of thyroid disease among women can be attributed to female sex hormones. ${ }^{13}$ Of these the most common age group was $36-45$ years accounting for $28.2 \%$ of cases. In a study by Dangi V and Meena RS in 2018 in Bhopal, India most cases were of age group 31-40 years. ${ }^{14}$ Among the 71 cases in our study, 13 cases $(18.3 \%)$ were of subclinical hypothyroidism while 58 cases $(81.7 \%)$ were of overt hypothyroidism which is in contrast with study by Aryal $\mathrm{M}$ et al which showed equal number of subclinical and overt hypothyroidism. ${ }^{11}$

In a study by Kumbhalkar et.al, most common symptom observed in hypothyroidism was weight gain followed by easy fatigability, dry skin, menstrual disturbance, constipation, depression, voice change and least of all neck swelling. ${ }^{15}$ Compared to this, in our study, the most common presenting complaint was constipation followed by weight gain. A reduction in gastrointestinal motility is responsible for constipation. ${ }^{16}$ In hypothyroidism there occurs a decreased basal metabolism and thermogenesis, an accumulation of hyaluronic acid and a decreased renal flow. These factors collectively lead to water retention and thus weight gain. ${ }^{17}$ Among other symptoms noted were lethargy, and dyspnea. Few cases presented with dry skin.

Our study revealed a normal echocardiography in 36.6\% of patients which is similar to the study done by Ramesh et.al which showed normal echocardiographic changes in $32.5 \% .{ }^{18}$ In a study by Al-Farttooosi et al., significant changes in ejection fraction, fractional shortening, changes in IVS thickness, IVS and LVPW ratio were found. ${ }^{19}$ In our study, $63.4 \%$ cases had abnormal echocardiographic changes. Two patients $(2.9 \%)$ had abnormal IVS/LVPW ratio, 6 patients $(8.4 \%)$ had pericardial effusion and 3 patients $(4.3 \%)$ had abnormal ejection fraction. Changes in diastolic interventricular septal thickness were seen among 13(18.3\%) cases.

The most common abnormality found was diastolic dysfunction which was seen among 31(43.1\%) patients; $28(90.3 \%)$ of them had impaired relaxation abnormality and $3(9.7 \%)$ had pseudonormal filling. Diastolic dysfunctionwassignificantlyhighinoverthypothyroidism as compared to subclinical hypothyroidism. All patients with $3(9.7 \%)$ with pseudonormal filling belonged to overt hypothyroidism. Shrivastava et al had found an abnormal echocardiography in $64 \%$ cases with the most common findings being diastolic dysfunction, which is very similar to our study. ${ }^{20}$ Diastolic dysfunction in hypothyroidism is due to decreased activity of sarcoplasmic reticulum calcium ATPase which is an enzyme that regulates intracellular calcium uptake. ${ }^{21,22}$ In a study by Behera et al. done in Eastern India, abnormal echocardiography findings were found in $67 \%$. There were diastolic dysfunction and pericardial effusion each 
Table 4: Association of Specific ECHO Changes in Relation to Types of Primary Hypothyroidism

\begin{tabular}{lllllll}
\hline $\begin{array}{l}\text { Echocardiographic } \\
\text { Changes }\end{array}$ & & Subclinical, n (\%) & Overt, n (\%) & Total & $\begin{array}{l}\text { Chi- } \\
\text { square }\end{array}$ & $\begin{array}{l}\text { p- } \\
\text { value }\end{array}$ \\
\hline Ejection Fraction & Normal & $11(84.6 \%)$ & $57(98.3 \%)$ & $68(95.8 \%)$ & 4.89 & 0.08 \\
Diastolic Inter & Abnormal & $2(15.4 \%)$ & $1(1.7 \%)$ & $3(4.2 \%)$ & & \\
ventricular septal & Normal & $10(76.9 \%)$ & $48(82.8 \%)$ & $58(81.7 \%)$ & 0.24 & 0.44 \\
thickness & Abnormal & $3(23.1 \%)$ & $10(17.2 \%)$ & $13(18.3 \%)$ & & \\
$\begin{array}{l}\text { Diastolic Left } \\
\text { ventricular posterior }\end{array}$ & Normal & $13(100 \%)$ & $52(89.7 \%)$ & $65(91.5 \%)$ & \multirow{2}{*}{0.46} & 0.28 \\
wall thickness & Abnormal & $0(0 \%)$ & $6(10.3 \%)$ & $6(8.5 \%)$ & & \\
IVS/LVPW Ratio & Normal & $13(100 \%)$ & $56(96.6 \%)$ & $69(97.2 \%)$ & & \\
& Abnormal & $0(0 \%)$ & $2(100 \%)$ & $2(2.8 \%)$ & & 0.66 \\
Pericardial Effusion & Present & $1(7.7 \%)$ & $5(8.6 \%)$ & $6(8.5 \%)$ & & \\
& Absent & $12(92.3 \%)$ & $53(91.4 \%)$ & $65(91.5 \%)$ & 0.012 & 0.69 \\
Diastolic & Present & $1(7.7 \%)$ & $30(51.7 \%)$ & $31(43.7 \%)$ & 8.37 & 0.004 \\
Dysfunction & Absent & $12(92.3 \%)$ & $28(48.3 \%)$ & $40(56.3 \%)$ & & \\
\hline
\end{tabular}

in $26.6 \%$ of cases..$^{23}$ Whereas in our study diastolic IVS thickness abnormality was the second most common abnormality after diastolic dysfunction. The cause for pericardial effusion in hypothyroidism is thought to be due to an increased capillary permeability and reduced lymphatic drainage from the pericardial space. ${ }^{24}$ Diastolic IVS thickness abnormality was found to be $18.3 \%$ in our study which is comparable with the study done by Preshant et al which showed $16.6 \% .{ }^{19}$ Diastolic left ventricular posterior wall thickness abnormality was about $8.5 \%$ in our study while in a study done by Mulki et.al it was $15.6 \%{ }^{25}$

In a study by Dangi $\mathrm{V}$ and Meena RS, $60 \%$ had abnormal echocardiography of which $32 \%$ had pericardial effusion, $18 \%$ had diastolic dysfunction and $4 \%$ has interventricular septum thickening. ${ }^{12}$ The deposition of mucopolysaccharides or edema in the cardiac wall is thought to alter the cardiac remodeling in hypothyroidism ${ }^{26}$. However, our study reported diastolic dysfunction as the most common abnormality followed by interventricular septum thickening and pericardial effusion. In our study, $8.5 \%$ of the patients had pericardial effusion whereas it was $18 \%$ in a study done by Sashikanth el.al. ${ }^{27}$ A meta-analysis done by Chen Xin et al. published in 2011 had concluded that hypothyroidism is significantly correlated with left ventricular diastolic dysfunction. However, no obvious correlation with left ventricular systolic dysfunction was found, suggesting that hypothyroidism leads to changes in cardiac function..$^{28}$ Our study also showed significant diastolic dysfunction.

This is a single hospital-based study, so the results obtained from this study cannot be generalized. No controls were recruited making it difficult to interpret whether findings were incidental or not. This is important regarding diastolic dysfunction as about $27 \%$ of normal population can have some degree of diastolic dysfunction. ${ }^{29}$ Comparing pre and post-treatment ECHO changes would have helped to better explain cardiac changes secondary to hypothyroidism.

\section{CONCLUSION}

We studied echocardiographic changes in newly diagnosed primary hypothyroidism patients with no known comorbidities. Commonest abnormality in our study was diastolic dysfunction. Other findings were diastolic IVS thickness abnormality, diastolic left ventricular posterior wall thickness abnormality, and pericardial effusion. Our study highlights the need for cardiac evaluation of patients with primary hypothyroidism so that early intervention can be performed to improve the clinical outcome.

\section{REFERENCES}

1. Vanderpump MPJ. Epidemiology of Thyroid Dysfunction - Hypothyroidism and Hyperthyroidism. Thyroid Int. 2009;2:1-12. https://doi.org/10.1093/bmb/ldr030 [Full text]

2. Joshi, Ansumali. (2018). Community based study of thyroid disorder prevalence in Nepal. Endocrine Abstracts (2019) 63 P776. https:// doi.org/10.1530/endoabs.63.P776 [Full Text]

3. Pinto A, Michael Glick. Management of Patients WithThyroidDisease:Oral HealthConsiderations. Journal of the American Dental Association 
2002: 133. https://doi.org/10.14219/jada. archive.2002.0299 [Pubmed]

4. Surks MI, Ortiz E, Daniels GH, et al. Subclinical Thyroid Disease: Scientific Review and Guidelines for Diagnosis and Management. Journal of the AmericanMedical Association. 2004;291(2):228238.https://doi.org/10.1001/jama.291.2.228 [Pubmed]

5. Klein I, Ojamaa K. Thyroid Hormone And The Cardiovascular System. NEJM 2001;344(7):501-509.https://doi.org/10.1056/ nejm200102153440707 [Pubmed]

6. Fommei E, Iervasi G. The role of thyroid hormone in blood pressure homeostasis: evidence from short-term hypothyroidism in humans. Journal of Clinical Endocrinology and Metabolism 2002;87(5):1996-2000. https:// doi.org/10.1210/jcem.87.5.8464 [Pubmed]

7. Udovcic M, Pena RH, Patham B, Tabatabai L, Kansara A. Hypothyroidism and the Heart. Methodist DeBakey Cardiovascular Journal 2017;13(2):55-59. https://doi.org/10.14797/ mdcj-13-2-55 [Pubmed]

8. Salvatore D, Davies TF, Brent GA. Williams Text Book Of Endocrinology. In: William Text Book of Endocrinology. 12th ed. Philadelphia: Elsveir Saunders; 2011:327-361.

9. Sitia S, Tomasoni L, Turiel M. Speckle tracking echocardiography: Anew approach to myocardial function. World J Cardiol. 2010;2(1):1-5 .https:// doi.org/10.4330/wjc.v2.i1.1 [PubMed]

10. Rodondi N, Bauer DC, Cappola AR. Subclinical thyroid dysfunction, cardiac fuction and the risk of heart faire. The cardiovascular Health study. J Am. Coll. Cardiol 2008;52(14):1152-1159. https://doi.org/10.1016/j.jacc.2008.07.009 [PubMed]

11. Aryal M, Gyawali P, Rajbhandari N, Aryal P, Pandeya D. A prevalence of thyroid dysfunction in Kathmandu University Hospital, Nepal. Biomedical Research. 2010;21:411-415. [Full Text]

12. Mahato R, Jha B, Singh $K$, Yadav B, Shah $S$, Lamsal M. Status of Thyroid Disorders in Central Nepal: A Tertiary Care Hospital Based Study. International Journal of Applied Sciences and Biotechnology 2015;3(1):119-122. https://doi. org/10.3126/ijasbt.v3i1.12218

13. Lauretta R, Sansone M, Sansone A, Romanelli F, Appetecchia M. Gender in Endocrine Diseases:
Role of Sex Gonadal Hormones. International journal of endocrinology, 2018, 4847376. https://doi.org/10.1155/2018/4847376

14. Dangi B, Meena R. Original Research Paper Medicine Study Of Cardiovascular Manifestation In Hypothyroidism Patients Admitted In Tertiary Care Center Hamidia Hospital Bhopal Indian Journal of Applied Research. 2018;(3):33-34. [DOI][Full Text]

15. Kumbhalkar SD, Choudhury SP. Echocardiographic evaluation of cardiac function in hypothyroidism. JMSCR Volume 05 Issue 08 August 2017. https://dx.doi.org/10.18535/ jmscr/v5i8.201

16. Shafer RB, Prentiss RA, Bond JH. Gastrointestinal transit in thyroid disease. Gastroenterology. 1984;86(5 Pt 1):852-5. PMID: 6706068. RíosPrego,

17. Anibarro LM, Sánchez-Sobrino, P. Relationship between thyroid dysfunction and body weight: a not so evident paradigm. International journal of general medicine, 2019; 12, 299-304. https:// doi.org/10.2147/IJGM.S206983

18. K. Ramesh K, Nayak BP. A study of cardiovascular involvement in Hypothyroidism. IAIM, 2016;3:74-80. [Full Text]

19. Minshed AJ Al- Farttoosi, Ghafour ASA, Shaker A, Zaidi A. Cardiovascular Manifestations of Primary Hypothyroidism. Iraqi Acadamy Scientific Journal. 2010;9(2):113-119. [DOI] [Full Text]

20. Shrivastava P, Tiwari A. ECG \& Echocardiographic Changes in Newly Diagnosed Primary Hypothyroidism. International Journal of Contemporary Medical Research 2017;4(3):607609. [Full Text]

21. Suko J. The calcium pump of cardiac sarcoplasmic reticulum. Functional alterations at different levels of thyroid state in rabbits. The Journal of physiology, 1973; 228(3), 563-582. https://doi. org/10.1113/jphysiol.1973.sp010100

22. Rodgers RL, Black S, Katz S, McNeill JH: Thyroidectomy of SHR: Effects on ventricular relaxation and on SR calcium uptake activity. Am J Physiol 1986;250:H861-H865 https://doi. org/10.1152/ajpheart.1986.250.5.H861

23. Behera B, Satpathy A, Samal K. Cardiovascular changes in newly detected hypothyroid patients in Eastern India. International Journal of Research in Medical Sciences 
September $20170 \mathrm{ct} ; 5(10): 4302-4306$. https://dx.doi.org/10.18203/2320-6012. ijrms20174171 [Pubmed]

24. Jabbar A, Pingitore A, Pearcs SH, Zaman A, Iervasi G, Razvi S.. Thyroid hormones and cardiovascular disease. Nat Rev Cardiol. 2017. January; 14 1: 39- 55. https://doi.org/10.1038/ nrcardio.2016.174 [PubMed] [ Google Scholar]

25. Mulki SR. Cardiovascular manifestation in hypothyroidism; Dissertation submitted to Rajiv Gandhi University of Health Sciences, Bangalore, Karnataka; 2011. [Full Text]

26. Mohandas M. Shenoy, Joel M. Goldman, Hypothyroid Echocardiographic

Cardiomyopathy:
Documentation of

Reversibility, The American Journal of the Medical Sciences, 1987,294: 1-9. https://doi. org/10.1097/00000441-198707000-00001.

27. M Shashikanth. Study of cardiac dysfuction in hypothyroidism: Indian Journal of Basic and Applied Medical Research. 2015; 4(2):111-116. [Full Text]

28. Chen Xin, Zhang Na, Zhang Wenliang, SHI Jingpu Meta-analysis of Doppler echocardiography in evaluating the relationship between subclinical hypothyroidism and left ventricular function[J]. Chinese Journal of Epidemiology, 2011,32(12);1269-1274. https://doi. org/10.1016/j.jjcc.2012.08.017 [PubMed] 